This is the peer reviewed version of Lees, J.M. (2006) "Behaviour of GFRP Adhesive Pipe Joints Subjected to Pressure and Axial Loadings", Composites, Part A: applied science and manufacturing v.37, pp. 1171-1179 which has been published on http://dx.doi.org/10.1016/j.compositesa.2005.05.033

\title{
Behaviour of GFRP adhesive pipe joints subjected to pressure and axial loadings
}

\author{
J.M. Lees, \\ University of Cambridge, \\ Department of Engineering, \\ Trumpington Street, \\ Cambridge, UK, CB2 1PZ \\ Tel: +44-1223-332678, \\ FAX: +44-1223-332662 \\ email: jmL2@eng.cam.ac.uk
}

\begin{abstract}
The corrosion resistance of glass-fibre reinforced polymers (GFRPs) is a key advantage in pipeline applications. Adhesive bonded joints are often used in tensile resistant GFRP pipe systems and the behaviour of small-diameter GFRP tubular pipe-sections joined by using an adhesive bonded coupler is of interest. In particular, the performance of the joint when subjected to a combination of high tensile axial loads and relatively low internal pressure is investigated. Experiments are conducted where a selfequilibrating pressurisation system is used to control the internal pressure and the coupled sections are tested in tension until failure. The cases of no pressure and a 32 bar working pressure are considered. A preliminary one-dimensional linear-elastic model is developed to predict the behaviour in the early stages of loading and to quantify the influence of internal pressure on the joint performance. Based on the experimental and analytical findings, it is concluded that the most critical case for the joint is likely to be the presence of large tensile loads in absence of internal pressure.
\end{abstract}

Keywords: Material: glass fibres, Property: strength, adhesive bonding, Testing: mechanical testing, Manufacturing/Processing: joints/joining, pipe 


\section{Introduction}

Much of our existing pipeline infrastructure is constructed from steel. Steel is strong, mechanically robust and relatively inexpensive. However, large sums of money are spent in trying to reduce the exposure of steel pipelines to corrosive environments. A recent study [1] indicated that in the United States alone the annual direct cost of corrosion in the gas-distribution network was \$5 billion US and the corresponding corrosion cost for drinking water and sewer systems was \$36 billion per year. One way to overcome this problem is to use a material with good corrosion resistance, such as glass fibre reinforced polymers (GFRPs), instead of steel. GFRPs represent an attractive alternative for pipelines subjected to severe internal or external environments in onshore or offshore applications.

One important issue in the design of GFRP pipe systems is the material anisotropy, which poses certain challenges that do not arise when dealing with an isotropic material like steel. For example, in filament wound GFRP pipes, the properties in the longitudinal and hoop directions will differ and be a function of factors such as the resin system, the fibre properties, the fibre volume fraction and the winding angle. For pipe applications, $\pm 55^{\circ}$ (with respect to the axial direction) fibre winding angles are commonly used. A further aspect in the design of any advanced composite system is the joint detail.

Pipe joining systems can be classified into one of two major categories depending on whether the joint is designed to take the full axial load due to the internal pressure (tensile resistant) or whether provision is made to resist the axial forces elsewhere on the pipeline system (non-tensile resistant). Adhesive joints are widely used as tensile resistant connections for GFRP filament-wound pipe sections. These joints are typically formed using either a bell and spigot system or a coupler to connect two pipe sections (see Fig. 1). It is important to note that, in the latter case, the anisotropic mechanical properties of the coupler can be quite different to those of the pipe since different fibre angles and processes are used during manufacture.

If a thin-walled pipe section is closed and fully pressurised then the hoop stresses will be twice the longitudinal stresses. However, there may well be cases where axial loads occur in the absence of 
pressure e.g. as a result of bending, axial loads due to thermal expansion, self-weight etc. and the performance under these conditions is also important. To improve our understanding of the interaction between internal pressure and additional tensile axial loads, the current work explores aspects of the performance of anisotropic GFRP adhesive-bonded coupled pipe joints when subjected to a variety of loading conditions. The investigation includes experimental results and a preliminary theoretical analysis of the behaviour in the linear-elastic stages of loading. While the particular focus is pipeline connections, many of the embodied principles are applicable to any adhesive-bonded advanced composite tubular joint subjected to a combination of pressure and axial load.

\section{Combined pressure/axial load testing of GFRP pipes}

There has been significant interest in the combined pressure/axial load testing of GFRP tubular members without a joint not only as a means of determining the pipe behaviour but also as a method of testing the biaxial properties of composites [2,3] In such tests, the stress ratios of hoop to axial loads are usually fixed, and the specimens are tested to failure. Work carried out by Soden et al [4] on lined or unlined (although only the lined results are referred to here), $100 \mathrm{~mm}$ internal diameter, $1 \mathrm{~mm}$ thick GFRP pipes with a $\pm 55^{\circ}$ winding angle considered stress ratios including pure tensile axial loading (0/1), ratios of 2/1 and 10/1 and various combinations in between. In the biaxial tests other than $2 / 1$, the specimens were loaded in tension or compression and the internal pressure applied simultaneously. The ends of the pipes were locally reinforced and end fittings had the dual function of providing a sealed pressurisation system and of applying load. As the axial load increased or decreased the pressure was carefully controlled to ensure the ratio of hoop to axial stress was kept constant. Pure hoop loading (1/0) and a range of axial compression loadings were also investigated. The highest axial stress could be carried at the 2/1 ratio; which is perhaps not surprising since this is the load condition for which the pipe winding angle had been optimised, and thus most of the load is taken by the fibres. In contrast, under pure tensile axial load (0/1) the failure stress was on average $70 \mathrm{MPa}$ and under pure hoop loading (1/0) the average failure stress was 583 MPa. A schematic failure envelope is shown in Fig. 2.

The focus of the current work was to consider the behaviour of joined tubular members under combined loadings and to explore cases where axial loads were applied independently from pressure. The case 
where longitudinal stresses were superimposed on stresses generated due to pressurisation was of most interest. Thus in contrast to much of the previous work on tubular pipes without joints, the pressure load was fixed and only the axial load varied, hence the ratio of the hoop stress to axial stress changed during testing.

A new testing procedure was developed. The loading system consisted of two components, a pressurisation system and a gripping system. The pressurisation rig was self-equilibrating and could be inserted inside the pipe to span the joint region. A schematic of the modular system may be found in Fig 3 . The pressurisation rig consisted of three stainless-steel sections with appropriate seals including two 100 mm long end pieces and a central insert, the dimensions of which could be varied depending on the joint length. The three sections were bolted together using a M30 bolt threaded at one end and different lengths of bolt could be used depending on the required length. Small holes were drilled through the GFRP pipe for the inlet and outlet valves which were used to control the pressure. The end grips were manufactured according to ASTM D 2105-97 [5] and are also shown schematically in Fig. 3. The basic grip configuration was a central wedge-shaped cone that reacted against inner and outer sleeves.

The advantages of the developed configuration for joint testing were that both the axial load and/or pressure could be readily adjusted independently. Furthermore, there was no initial requirement for additional reinforcement of the ends of the pipe so the specimens were easy to prepare. However, it is important to note that there is a region of pipe at the end of the pressurisation rig which is unpressurised thus the maximum applied axial load will be limited by the pipe capacity in this region (see Fig. 2). If the specimens fail in the joint region at a load less than the axial capacity of the pipe, this is not a major limitation. However, if this is a critical factor (and indeed one of the joints reported here did fail in this manner) the end regions of the pipe need to be reinforced and/or ways to extend the pressurisation system to react against the collets of the end grips could be investigated. Indeed, the development of a simple and efficient pressurisation system that encompasses the length of the pipe would be a useful improvement as a wider range of pipe and coupler systems could then be considered without being limited by the unpressurised axial pipe capacity. 


\section{Experimental work}

The pipes considered in the experimental programme are commercially available $80 \mathrm{~mm}$ ID, GFRP filament-wound pipes with 52 $\pm 7 \%$ glass content (by volume) and a winding angle of $\pm 55^{\circ}$. The stated minimum pipe-wall thickness is $3.2 \mathrm{~mm}$ but the measured wall thicknesses are often greater (up to $4.5 \mathrm{~mm}$ ). The manufacturers' stated properties [6] in the axial and hoop directions are an ultimate tensile stress of $\sigma_{u x}=75 \mathrm{MPa}$ and $\sigma_{u \theta}=210 \mathrm{MPa}$ and tensile moduli of $E_{x}=12 \mathrm{GPa}$ and $E_{\theta}=20.5 \mathrm{GPa}$ respectively. The shear modulus $G_{x}$ is $11.5 \mathrm{GPa}$ and the Poisson's ratios relating the axial deformation due to a hoop loading and the hoop deformation due to an axial load are $v_{x \theta}=0.65$ and $v_{\theta x}=0.38$ respectively. The coupler supplied by the same pipe manufacturer is $91 \mathrm{~mm}$ long, around 6.8 mm thick with a $10 \mathrm{~mm}$ central stop and an inside diameter of approximately $85.8 \mathrm{~mm}$. The fibre layout in the coupler differs from that of the pipe and the values supplied by the manufacturer for the coupler material are $\sigma_{u x}=40 \mathrm{MPa}, \sigma_{u \theta}=20 \mathrm{MPa}, E_{x}=18 \mathrm{GPa}, E_{\theta}=20 \mathrm{GPa}, v_{\theta x}=0.12$ and $v_{x}=0.11$.

In order to make a connection between two lengths of pipe, the ends of the pipe sections are shaved along the bonded length to a reduced wall thickness of $2.9 \mathrm{~mm}$, leaving an outside diameter of $85.8 \mathrm{~mm}$. In addition, the inner surface of the coupler is abraded, removing a further 0.1-0.3 mm of material. To produce a joint, adhesive is applied to the shaved pipe ends and the coupler. Each end of pipe is then inserted into the coupler. The resulting adhesive bond line thickness is between 0.1 and $0.3 \mathrm{~mm}$. The joint is then heat cured in accordance with the manufacturer's recommendations [7].

When required, the pressurisation system is inserted into the pipe bore over the joint region. The end grips are attached to the pipe and the joined specimen is placed in an Instron testing machine. For the pressurised tests, the annulus between the pressurisation rig and the pipe bore is filled with hydraulic oil and the specimens are pressurised to the required level. The applied tensile load is increased at a rate of $5 \mathrm{~mm} / \mathrm{min}$. As the tensile load increased, the internal pressure increases due to the Poisson's ratio effects causing a contraction of the pipe. Thus, the outlet valve is used to release the pressure at stages during testing to maintain the chosen pressure. Although the process could be controlled automatically, due to equipment limitations it was controlled manually and, as a result, small pressure variations were apparent (differences of approximately 5\% were noted). 
The test series consisted of two phases. In the first phase, a total of five joints with either a $20 \mathrm{~mm}$ or $40 \mathrm{~mm}$ bond length with an internal pressure of 0 bar or 32 bar were tested to failure in tension. The joints with a $20 \mathrm{~mm}$ bond length were prepared by removing $20 \mathrm{~mm}$ from either end of the coupler and each of the pipe sections to give an overall coupled length of $50 \mathrm{~mm}$ (the aim was to purposely produce a lower strength joint to enable a wider comparison of results). In the second phase, detailed strain measurements of a joint with a $40 \mathrm{~mm}$ bond length (coupled length of approximately $90 \mathrm{~mm}$ ) were undertaken.

\subsection{Phase 1 - Tension/pressure tests}

When no internal pressure was applied, a pipe specimen with a coupler and a $40 \mathrm{~mm}$ lap length failed due to debonding along one side of the joint at a load of $74.4 \mathrm{kN}$. The corresponding value for a $20 \mathrm{~mm}$ joint was $58.0 \mathrm{kN}$. The tensile capacity of the joints tended to increase somewhat with increasing pressure and the corresponding failure loads for an internal pressure of 32 bar were $70.6 \mathrm{kN}$ for the $20 \mathrm{~mm}$ lap and $77.5 \mathrm{kN}$ for the $40 \mathrm{~mm}$ lap. Both these specimens failed due to debonding in the joint. However, a second $40 \mathrm{~mm}$ lap joint subjected to 32 bar pressure failed in the pipe section. No leakage of the oil was noted throughout the tests. For the pressurised joints, the ratio of the applied hoop to axial stress at failure was much lower than what the joint would be subjected to under restrained pressurisation conditions (2/1). The tests give insight into how the joint would behave under such conditions and, in particular, the results suggest that the presence of pressure is potentially beneficial in terms of the tensile joint capacity.

\subsection{Phase 2 - Strain measurements}

Detailed measurements of a pipe with a $40 \mathrm{~mm}$ joint were taken using a series of strain gauges mounted on both the inside and outside of the pipe and joint sections (see Figs 4 and 5). The wires from the inner gauges were fed through a sealed hole in the pressurisation rig. The notation used to identify a particular strain gauge is a combination of one number and a letter. The number corresponds to the gridline position along the $\mathrm{x}$-axis (see Fig. 4) and the letter denotes whether the gauge is on the outer $(\mathrm{O})$ or inner $(\mathrm{I})$ surface. At gridlines 1 and 5 the inner and outer gauges were closely aligned however, due to the difficulty in positioning the inner gauges, slight offsets between the inner and outer gauges were noted at gridlines 3 and 4 and 7 and 8 . Hoop and longitudinal displacement gauges were also used to monitor the 
extensions on the pipe, coupler and at the pipe coupler interface (see Fig. 5) but these results are not reported here.

Two tests were carried out on the same specimen. The first was a tension-only test, in which the specimen was loaded up to $25 \mathrm{KN}$ and then unloaded to $0 \mathrm{kN}$. The specimen remained in the rig (and was thus partially restrained longitudinally) and was pressurised over a length of $350 \mathrm{~mm}$ to approximately 32 bar and then tested in tension. The strain results from these tests are discussed in a later section.

\section{Analytical work}

The pipe and coupled regions were considered to be thin-walled elastic orthotropic beams with different thicknesses (see Fig. 6). The adhesive interface was assumed to be fully bonded and was not taken into account explicitly. The approach was to consider what happens due to the pressure acting on the beams and then consider what happens due to the axial load. The principle of superposition could then be used to combine the load cases.

The elastic beams were modelled using the method outlined in Calladine [8] with appropriate substitutions to account for the GFRP material orthotrophy. A cylindrical element of length $d x$ subtending an angle $d \theta$ is shown in Fig. 7. The element is subjected to in-plane stress-resultants $N_{\theta}$ and $N_{x}$ and bending moments $M_{x}$ and $M_{\theta}$. A pressure $p$ is applied to the cylinder of radius $a$ and thickness $t$. The radial displacement is denoted as $w$ and is positive outwards. The axial stress-resultant, $N_{x}$, is assumed to be constant and, in the first instance, will be taken to be zero.

Classical small-deflection elastic axisymmetric thin-shell equations give:

$$
D \frac{d^{4} w}{d x^{4}}+k w=p
$$

where 
$k=\frac{E_{\theta} t}{a^{2}}$

and $D$ is the flexural rigidity of the beam.

For an orthotropic material in plane stress, the relationships between the axial strain, $\varepsilon_{x}$, hoop strain, $\varepsilon_{\theta}$, and shear strain, $\gamma_{x \theta}$, and axial stress, $\sigma_{x}$, hoop stress, $\sigma_{\theta}$, and shear stress, $\tau_{x \theta}$, are as follows:

$\left[\begin{array}{c}\varepsilon_{X} \\ \varepsilon_{\theta} \\ \gamma_{x} \theta\end{array}\right]=\left[\begin{array}{ccc}\frac{1}{E_{X}} & -\frac{v_{x} \theta}{E_{\theta}} & 0 \\ -\frac{v_{\theta x}}{E_{X}} & \frac{1}{E_{\theta}} & 0 \\ 0 & 0 & \frac{1}{G_{x} \theta}\end{array}\right]\left[\begin{array}{c}\sigma_{x} \\ \sigma_{\theta} \\ \tau_{x \theta}\end{array}\right]$

The stress $\sigma_{x}$ can be integrated over the section thickness to find the moments where:

$M_{X}=\left(\frac{E_{X}}{1-v_{x} \theta^{v} \theta x}\right) \frac{t^{3}}{12} \kappa_{X}=D \kappa_{X}$

and thus for an orthotropic material:

$D=\left(\frac{E_{x}}{1-v_{x} \theta^{v} \theta x}\right) \frac{t^{3}}{12}$

The form of eqn 1 is analogous to the governing equation of a beam on an elastic foundation presented by Hetényi [9] (although a different notation is used) and the pipe hoop stiffness acts like the springs in an elastic foundation [10].

The solution of the equation is of the form $w=e^{m x}$ which leads to:

$w=e^{\lambda x}\left(C_{1} \cos \lambda x+C_{2} \sin \lambda x\right)+e^{-\lambda x}\left(C_{3} \cos \lambda x+C_{4} \sin \lambda x\right)$

where

$\lambda=\sqrt[4]{\frac{k}{4 D}}=\sqrt[4]{\frac{E_{\theta} 3\left(1-v_{x} \theta^{v} \theta x\right.}{a^{2} E_{x} t^{2}}}$ 
Thus if the geometry and material properties are known, $\lambda$ can be determined for the pipe and the coupled region. For the pipe, the manufacturer's properties were used. However, the coupled region consists of a bonded section of pipe and coupler. In this case the law of mixtures was used (again using manufacturer's values) to find the equivalent properties for the joined region.

It is of note that the characteristic lengths of both the pipe $(\sim 20)$ and the coupler $(\sim 3)$ sections are relatively long (e.g. $\lambda l \sim>\pi)$, hence both the pipe and coupler can be considered as semi-infinite without an undue loss of accuracy. As a result, only two constants are required in eqn 6 which can be determined by considering the appropriate boundary conditions. Once an expression for $w$ has been found, the rotation $(d w / d x)$, moment and shear stress resultants can all be determined by taking the appropriate derivatives.

The pressure is applied as a uniform load over the length of the pressurised region. In the first instance, the axial load is applied as a unit contraction and included using Poisson's ratio effects leading to:

$$
w=-\frac{v_{\theta X} N_{X}}{2 \pi a t E_{X}}
$$

As the model is a line element, the moment due to the load eccentricity is modelled explicitly. However, any additional moment due to the axial force acting on an element in the deformed shape is not taken into account. This will make some difference to the results but it is expected that the axial contribution to the bending stresses will not be large for the pipe sections considered in the current work.

The pressure and axial loads are applied to the coupled and pipe sections separately. The relevant cases are superimposed and then compatibility is used to ensure continuity between the two beam sections. The loads and reactions to ensure continuity are summarised in Table 1 . Because of the symmetry of the problem, only one half of the joined section is considered. Using the analysis presented above, a MATLAB [11] programme was written so that different pressure and axial load combinations could be considered. 


\section{Discussion}

Selected experimental strain gauge readings on the joined specimen for the tension-only test (up to $25 \mathrm{kN}$ ) and the pressure/tension test readings over a similar range present an opportunity to compare the predicted strains from the simplified model with those determined experimentally (note the same specimen was used for both tests). The longitudinal and circumferential strains for the no-pressure case and the 32 bar case are shown in Figs 8 and 9 respectively. For clarity, there are two plots for the circumferential gauges where in (a) selected gauges on the pipe section (gridlines 1,3 and 4) and in (b) selected gauges on the coupled section (gridlines 5 and 7-9) are indicated. The predicted results are denoted by the gridline reference followed by the letter P.

It is of note that during pressurisation, some restraint was provided by the grips where the axial load increased to a value of approximately $5 \mathrm{kN}$ during this process (see Fig. 9(a) for a schematic of the loading sequence). As the exact level of restraint is unknown, from the experimental load and pressure readings, an expression for the change in pressure with increasing axial load was determined. These were included in the model to predict the strain behaviour during pressurisation e.g. the longitudinal strain due to Poisson's ratio effects could then be subtracted from the strains due to the axial load. The internal pressure acts to reduce the longitudinal tensile stresses and increase the circumferential tensile stresses.

From Figs 8 and 9 it can be noted that while in some cases the absolute values differ, the general trends are predicted fairly well by the model.

A certain variability in the strain gauge readings is evident from the longitudinal readings where the gauges in similar locations on the pipe but on opposite ends (gridline 1 vs 10) give different results (Figs 8(c) and 9(c)). From the circumferential results, differences between the strain readings on the inner and outer surfaces of the pipe are noticeable where the strains on the inner surfaces are higher than those in a similar location on the outer surface. For gridline 1 (on the pipe) the difference is not so great and would indicate that the assumption of thin-walled behaviour is reasonable. For gridline 5 in the coupled region, the difference is greater. However, as the coupled region is a thicker section, thin-walled theory may be less appropriate. 
The analysis assumes asymmetric behaviour with no twist and the readings from the strain rosettes were checked to confirm the validity of this assumption. Under these conditions, it would be expected that the strain at $45^{\circ}$ should be the mean of the longitudinal and hoop strains. Although small deviations were noted, for the most part this was indeed the case. A further inherent assumption in the proposed elastic model is that the pipe and coupler are fully bonded throughout the coupled region. If the bending of the GFRP is neglected, Volkersen's model (with certain modifications to account for a tubular geometry [12]) can be used to give an initial indication of the shear transfer between the pipe and coupler. Such an approach would suggest that the majority of the load is transferred in the first $10 \mathrm{~mm}$ from the edge of the joint. However, for full details of the stress transfer in these regions, a more detailed analysis would be required.

The predicted radial displacements along the length for both the unpressurised and the pressurised cases are noted in Fig. 10. Close to the end of the coupled region there are very high changes in deformation suggesting that the results will be sensitive to any small variation in the position of the strain gauge. Also the strain gauges have a given gauge length so the readings will necessarily be an average. The predicted curves also suggest that the assumption of semi-infinite behaviour is not unreasonable since the end boundary conditions do not seem to influence the results in the regions of most interest.

Because of the smaller thickness and the large Poisson's ratio of the filament wound pipes it can be seen in Fig. 10 that, under tensile load, the pipe contracts more than the coupler and thus tends to pull away from the adhesive. When pressurised, this effect is less severe since the pressure acts to push the pipe against the coupler so it is only under higher axial loads that significant peel stresses develop. The existence of through-thickness compression may also enhance the Mode I joint performance.

Selected predicted and experimental results for the combined pressure/tension case up to a load of $65 \mathrm{kN}$ are shown in Fig. 11. The results compare fairly well until a load of about $50 \mathrm{kN}$ at which point they diverge. Of particular note are the circumferential gauges on gridlines 2, 4 and 7 and the longitudinal gauge on gridline 5 which all show a decrease in tensile strain at around $50 \mathrm{kN}$. In contrast, the circumferential tension on gridline 5 increases. These results are consistent with slip taking place at the 
end of the joint between the pipe and the coupler. The occurrence of slip was also noted in the hoop gauge reading between the ends of the pipe and coupler (see Fig. 5) and a cracking noise was heard during testing. The proposed predictive model breaks down for two reasons, the elastic non-linearity in the pipe and coupler behaviour and also due to any damage or fracture events. While elastic non-linearity could be easily incorporated into the model, it is not clear that an extension of a line element model would be most appropriate for the post fracture regime as a detailed analysis of the load distribution through the joint region is required. Nevertheless the model gives insight of the behaviour up to first fracture and helps identify the interactions that occur in the linear elastic region.

The elastic model indicates that for the loading, pipe and coupler parameters under consideration, the tensile peel stresses in the joint region will be higher for the non-pressurised rather than the pressurised case. This would suggest that first fracture will occur at a lower load for the non-pressurised case and this may help to explain the experimental trend where the ultimate tensile capacity increased somewhat with increasing pressure.

Also of note is that for the coupled section there are two transfer zones, one from the pipe to the pipe/coupler region and the second from the pipe/coupler region to the coupler where there is a central coupler region of $10 \mathrm{~mm}$ length (see Fig. 4). Although only the results at the pipe/coupler end have been developed here, the same approach can be used at the pipe end. However, the difference in the material properties between the joined section and the coupler-only sections are not as significant as between the pipe and joined section so the bending effects may not be as great. There is the further complication in the region of the adhesive fillet which was not explicitly included in the model.

The assumed material properties used in the model were those given by the manufacturers; but in practice, these properties may vary. The quality of the adhesive bonding, the size of the adhesive fillet, the bondline thickness etc. may also vary from joint to joint. Further tests are required to confirm the experimental findings.

While the focus of much of the work presented here has been the initial elastic behaviour, the study highlights the importance of considering the performance of the joined system under a variety of loading 
conditions. Failure loads obtained from experiments where the design capacity of the joint is determined on the basis of a (2/1) ratio of the hoop and longitudinal stresses may be unconservative when filamentwound pipes are subjected to high tensile loadings and low pressure conditions. Although not considered here, the case of external pressure or an internal suction developing in the pipe would again be expected to further influence the joint capacity. The sensitivity of the system will depend on the relevant material properties of the pipe and coupler sections; and different filament winding angles, pipe thicknesses etc. will give different results. Areas for future work would include more extensive experiments on joined specimens under a wider range of load combinations and a detailed analysis of the fracture behaviour at failure.

\section{Conclusions}

The GFRP filament-wound adhesive joint behaviour has been found to depend on the combined pressure/tension loading. An elastic one-dimensional analysis using orthotropic material properties was found to give a reasonable prediction of the joined behaviour in the early elastic stages of loading up until slip occurred between the coupler and the pipe. From the initial experimental and analytical findings, it is concluded that initial damage is more likely to occur when the joint is subjected to large tensile loads in the absence of internal pressure. Further work is required to confirm the experimental results and to model the later stages of behaviour.

\section{Acknowledgements}

The author wishes to thank Mr. Roger Denston for conducting the strain experiments described in this paper. She is also grateful to Allan McRobie and Andrew Palmer for their support. This work was in part funded under EPSRC grant GR/R09770.

\section{References}

[1] Koch GH, Brongers MPH, Thompson NG, Virmani YP, Payer JH. Corrosion Costs And Preventive Strategies In The United States. Office of Infrastructure Research and Development, Report FHWA-RD01-156, 2002. 
[2] Chen AS, Matthews FL. A review of multiaxial/biaxial loading tests for composite materials. Composites 1993; 24(5): 395-406

[3] Soden PD, Hinton MJ, Kaddour AS. Biaxial test results for strength and deformation of a range of Eglass and carbon fibre reinforced composite laminates: failure exercise benchmark data. Composites Science and Technology 2002; v 62: 1489-1514.

[4] Soden PD, Kitching R, Tse PC. Experimental failure stresses for $\pm 55^{\circ}$ filament wound glass fibre reinforced plastic tubes under biaxial loads. Composites 1989; 20(2): 125-135.

[5] ASTM D 2105-97. D2105-97 Standard Test Method for Longitudinal Tensile Properties of "Fiberglass" (Glass-Fiber-Reinforced Thermosetting-Resin) Pipe and Tube. ASTM. 1997.

[6] Wavistrong Epoxy Pipe Systems, Engineering Guide for Wavistrong Filament Wound Epoxy Pipe Systems. Future Pipe Industries B.V. Hardenberg, Netherlands. 1997.

[7] Wavistrong EASY-FIT adhesive, Adhesive-bonded jointing systems - Instructions. Future Pipe Industries B.V. Hardenberg, Netherlands. Rep 419 Rev 00-0101.

[8] Calladine CR. Theory of Shell Structures. Cambridge University Press, Cambridge 1983.

[9] Hetényi M. Beams on Elastic Foundation: Theory with Applications in the Fields of Civil and Mechanical Engineering. University of Michigan Studies Scientific Series XVI, 1961

[10] Lees JM. Combined pressure/tension behaviour of adhesive-bonded GFRP pipe joints. In Hollaway

LC, Chryssanthopoulos MK, Moy SSJ, Eds Advanced Polymer Composites for Structural Applications in Construction, ACIC 2004, Woodhead Publishing Ltd. p. 377-383.

[11] The Mathworks Inc. MATLAB Version 6.1.0.450, Release 12.1 2001.

[12] Pickett AK, Hollaway L. The analysis of elastic adhesive stresses in bonded lap joints in FRP structures. Composites Structures 1985; 3: 55-79. 
Table 1. Beam models and load cases

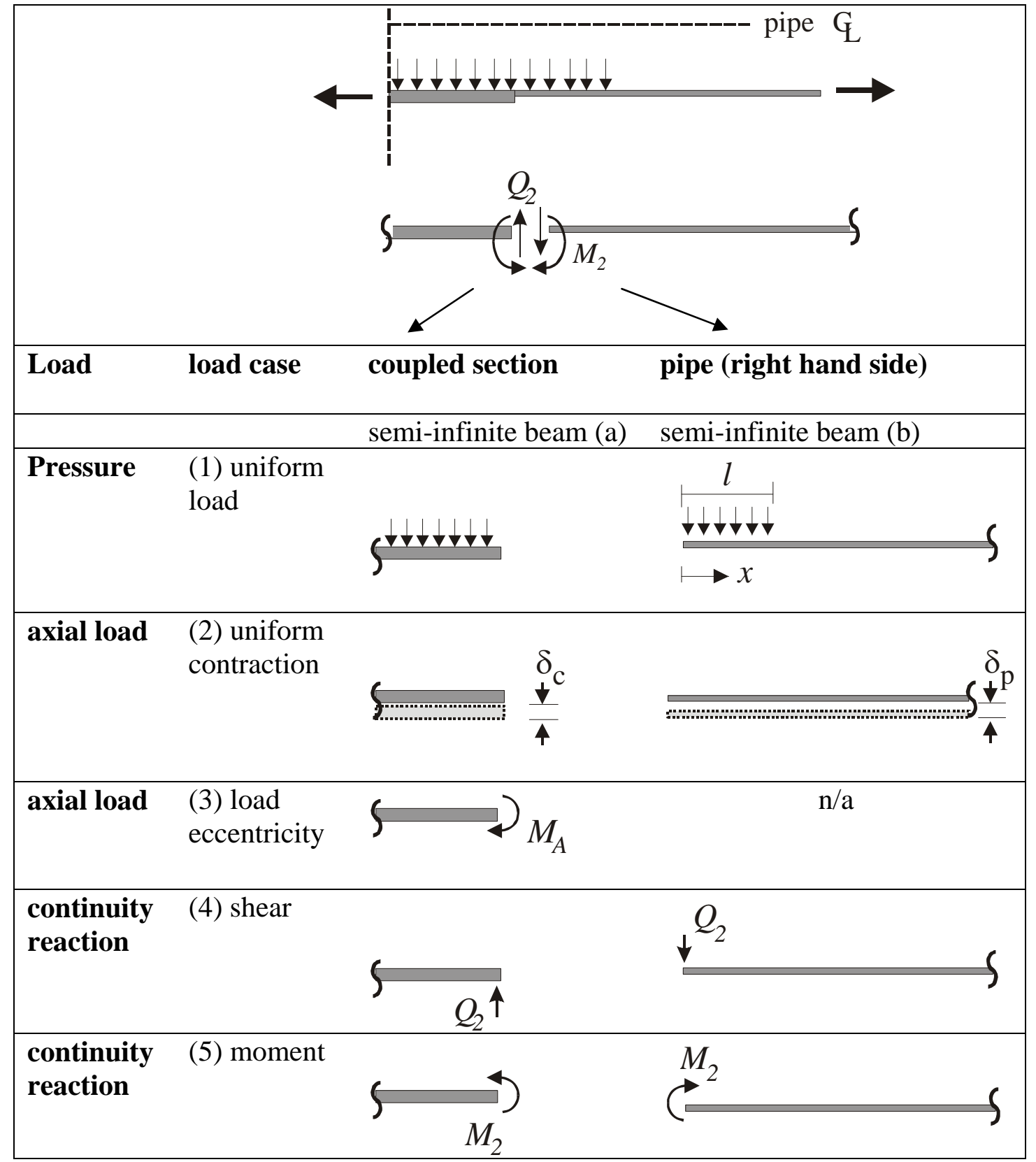




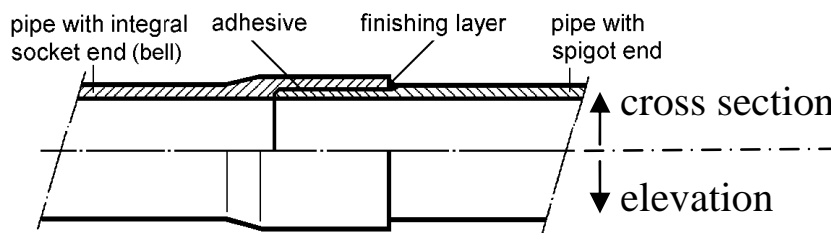

(a)

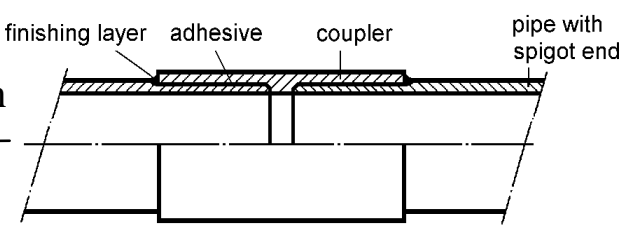

(b)

Figure 1. Pipe joints (a) bell and spigot and (b) coupler

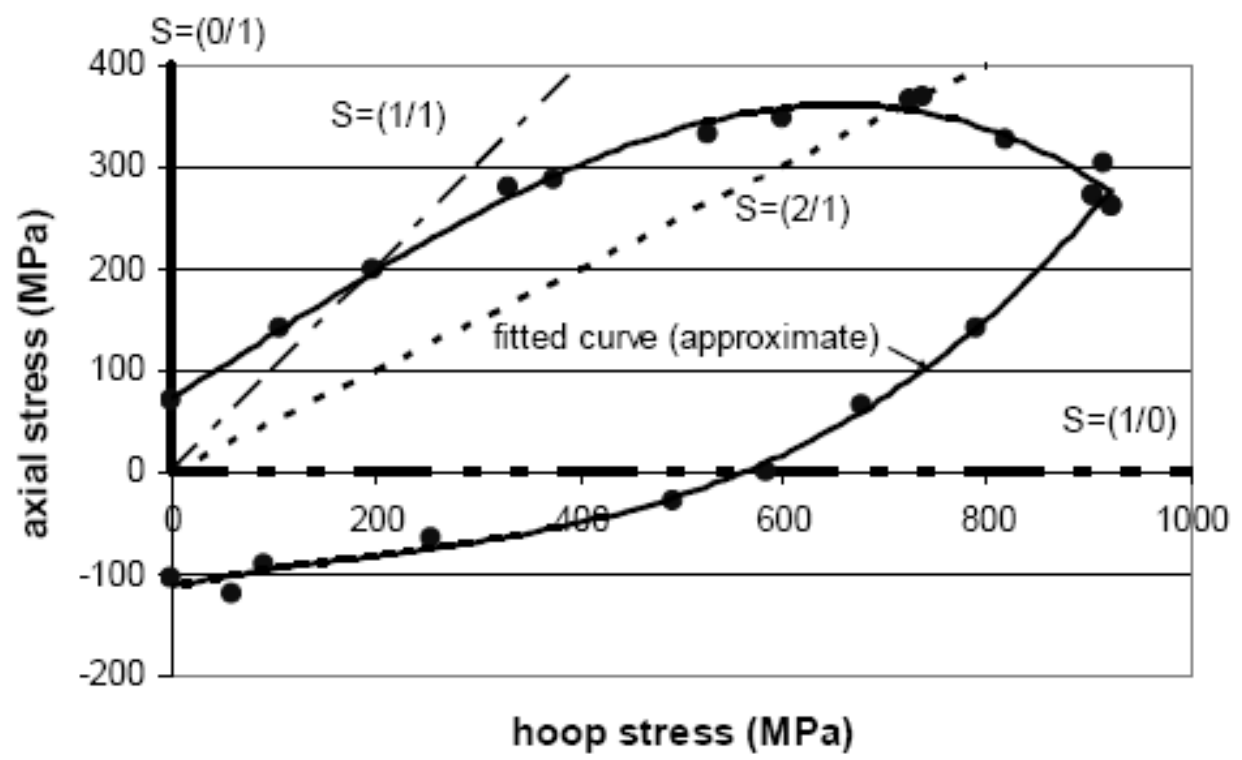

Figure 2. Schematic tubular pipe failure envelope (after [4])

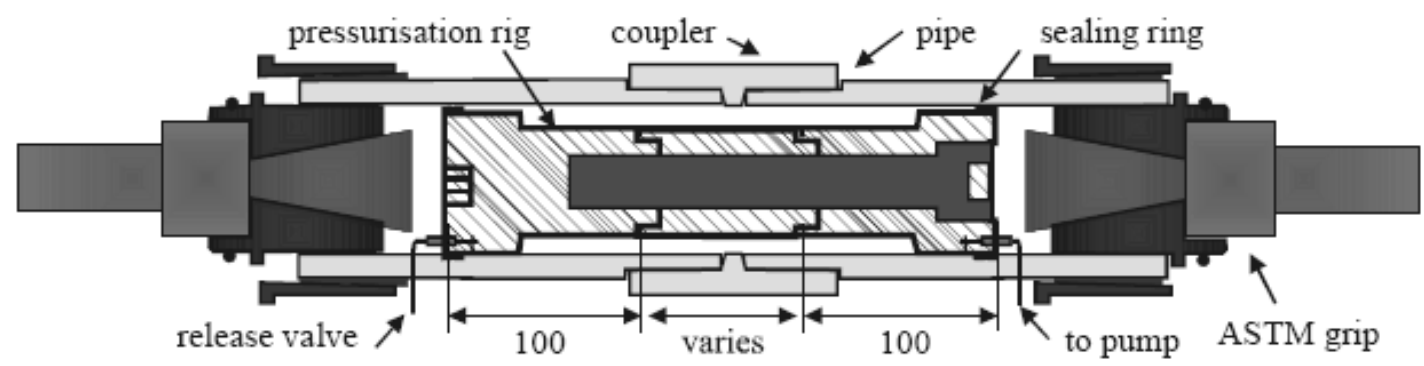

Figure 3. Schematic section through pipe and coupler showing self-equilibrating pressurisation system and tensile grips 


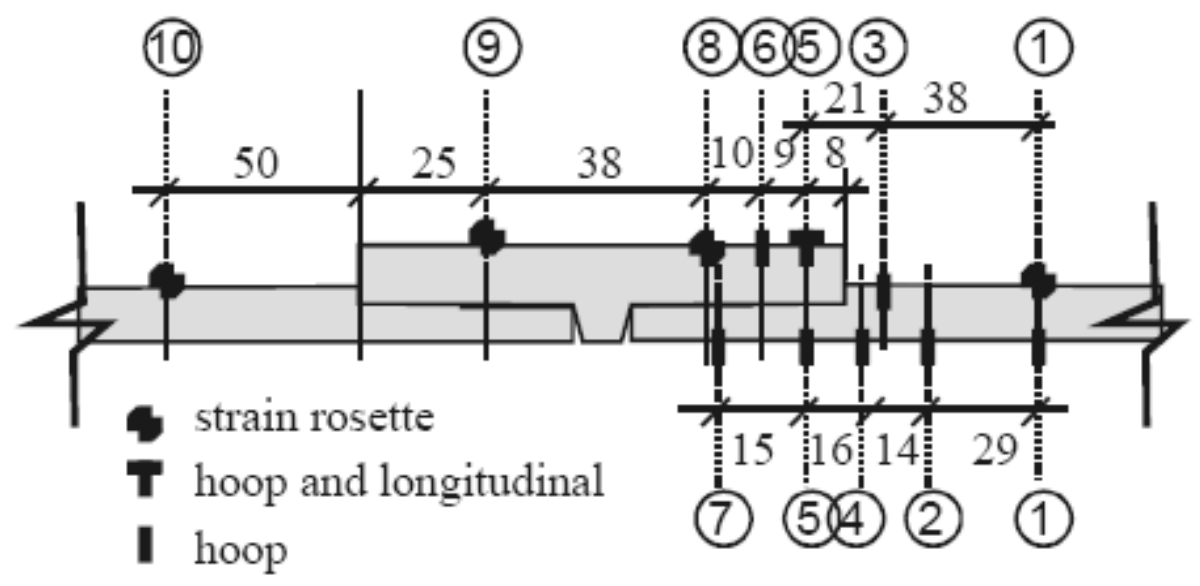

Figure 4. Strain gauge locations on joined specimen

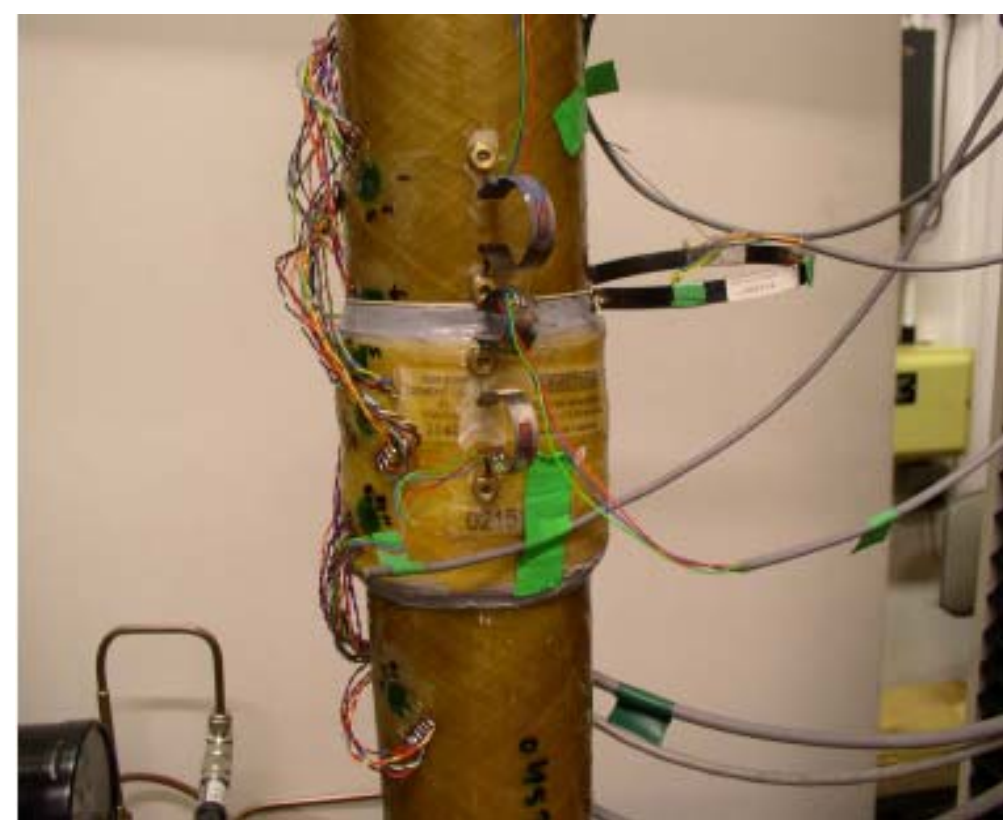

Figure 5. Pipe joint under testing

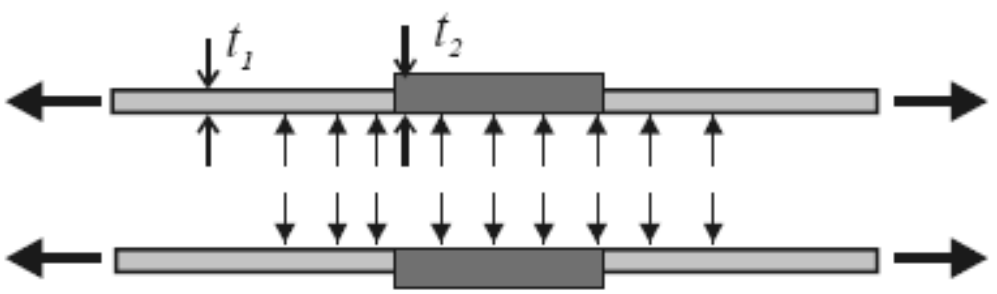

Figure 6. Beam model 


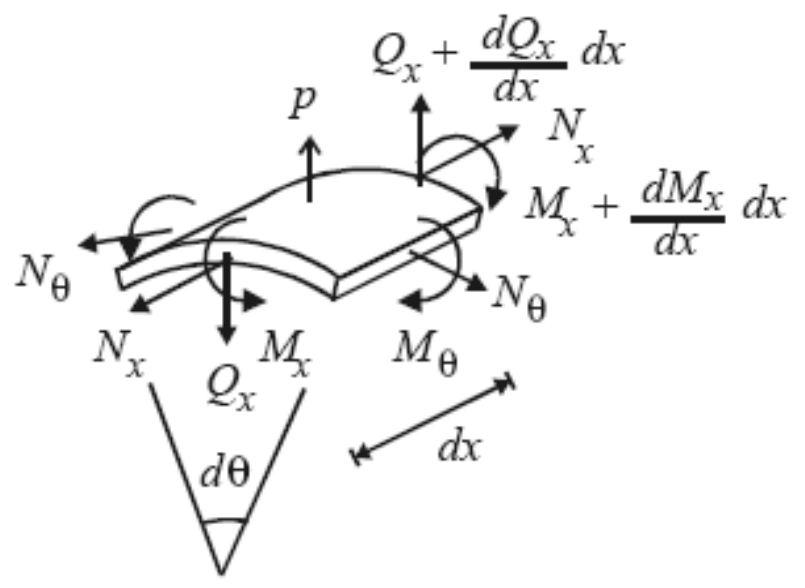

Figure 7. Cylindrical element notation 


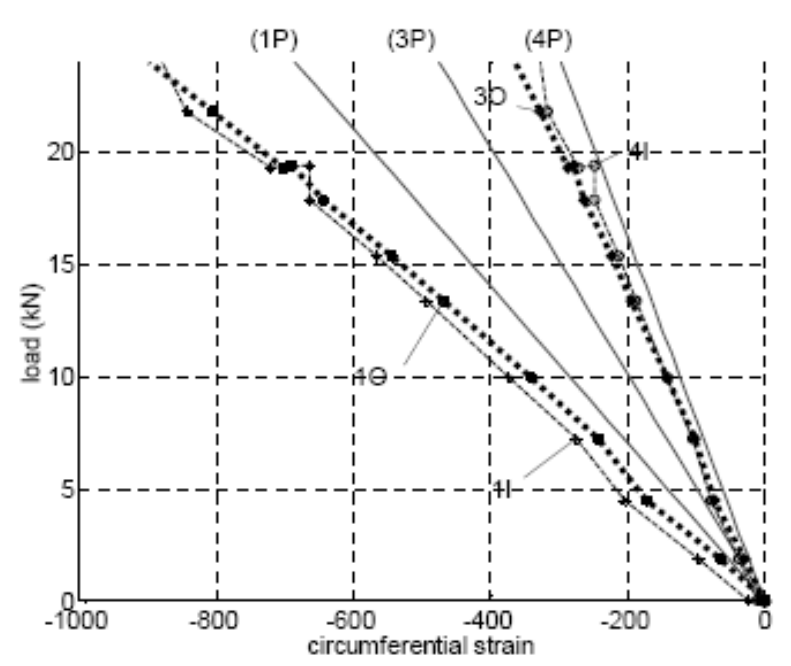

(a)

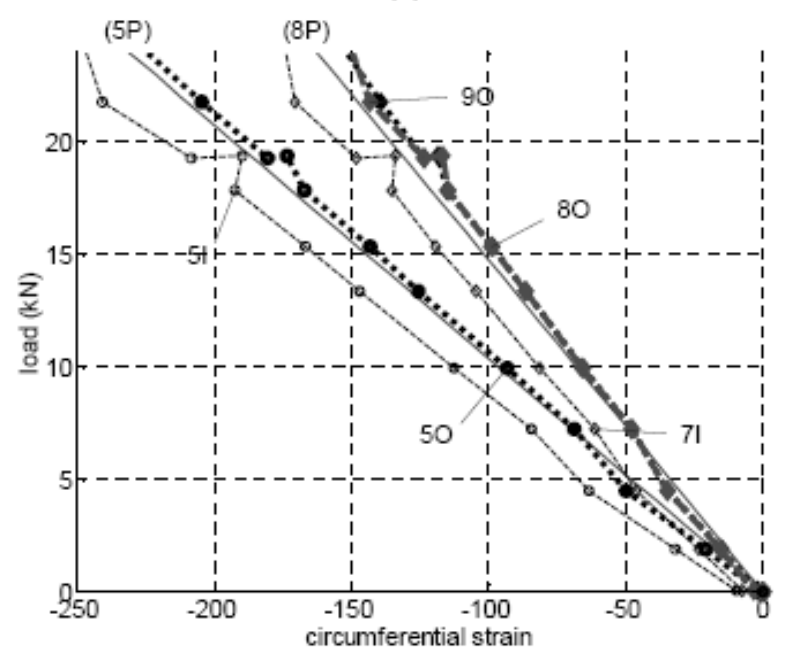

(b)

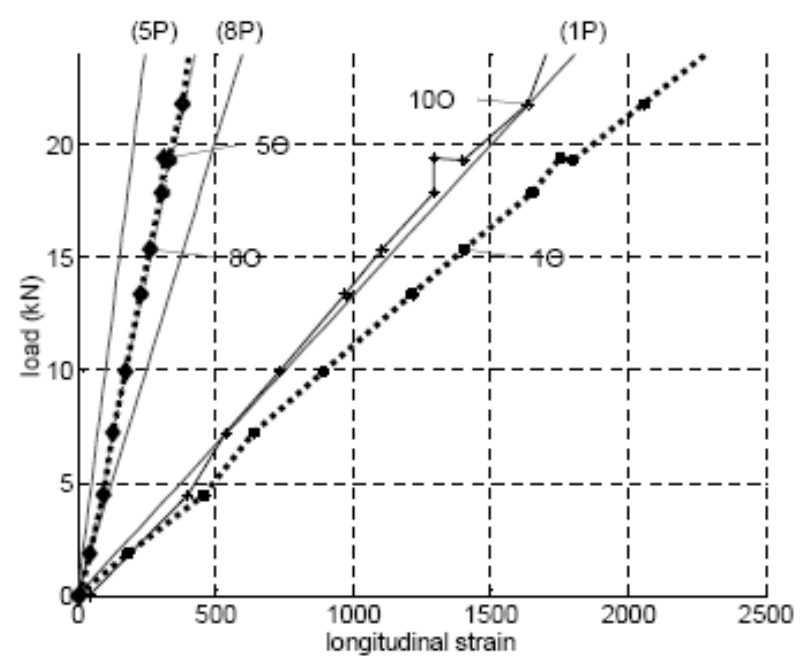

(c)

Figure 8. Comparison of experimental and theoretical strains for tension test (between a load of 0 and $25 \mathrm{kN}$ ) with no internal pressure (a) circumferential strains in pipe region (b) circumferential strains in coupled region and (c) longitudinal strains. See Fig. 4 for strain-gauge locations. 


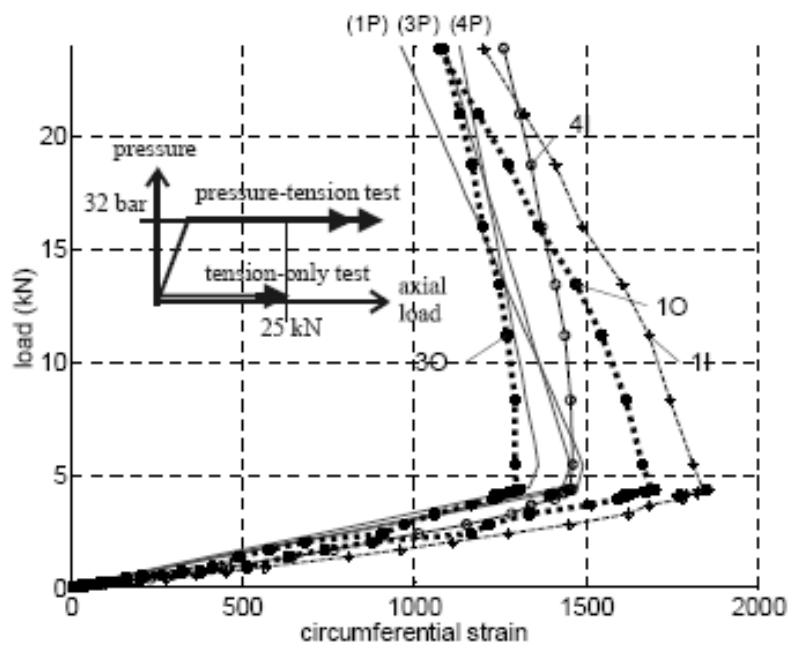

(a)

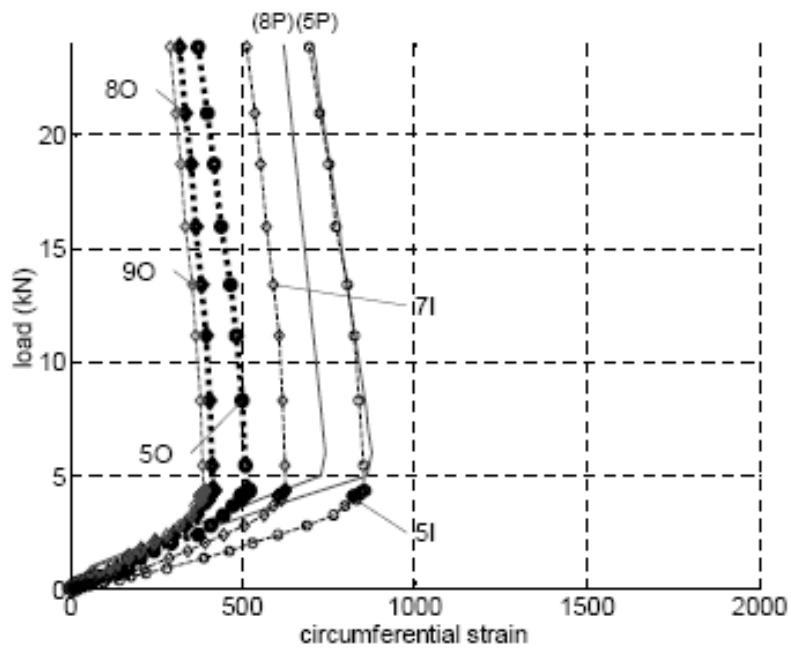

(b)

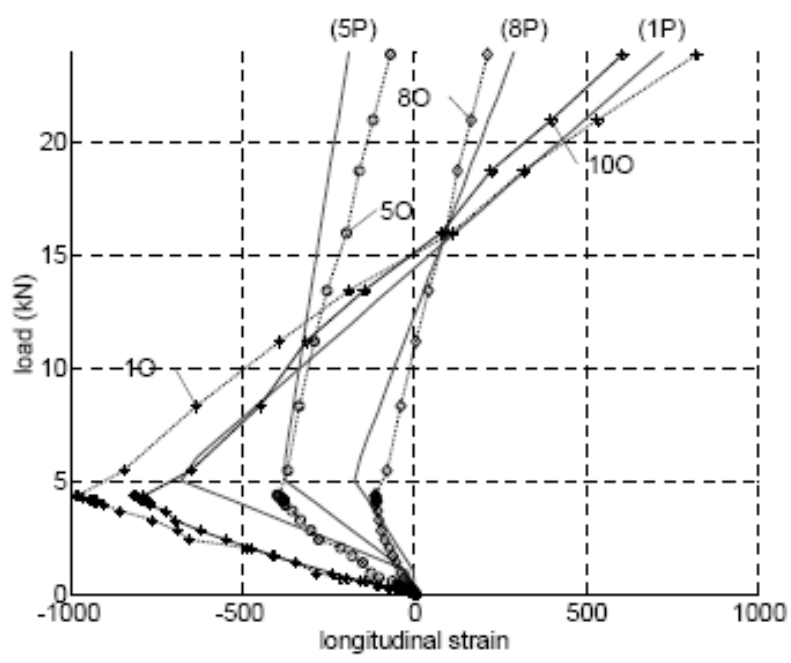

(c)

Figure 9. Comparison of experimental and theoretical strains for pressure/tension test (between a load of 0 and $25 \mathrm{kN}$ and with 32 bar internal pressure) (a) circumferential strains in pipe region (b) circumferential strains in coupled region and (c) longitudinal strains. See Fig. 4 for strain gauge locations. 


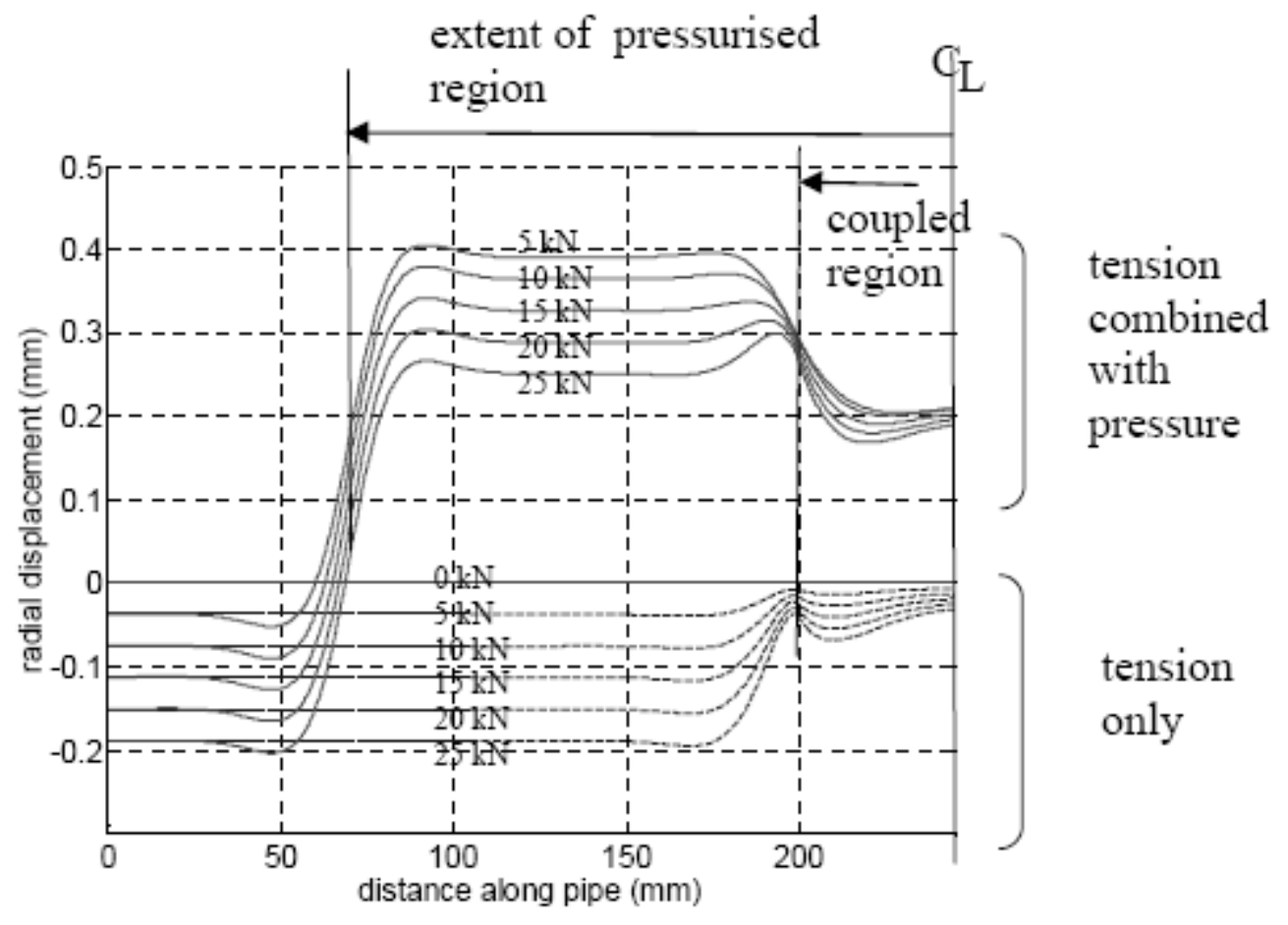

Figure 10. Comparison of tension-only and pressure/tension behaviour - predicted radial displacement along length. Lower curves are for tension-only; upper curves are for tension combined with a pressure of 32 bar. 


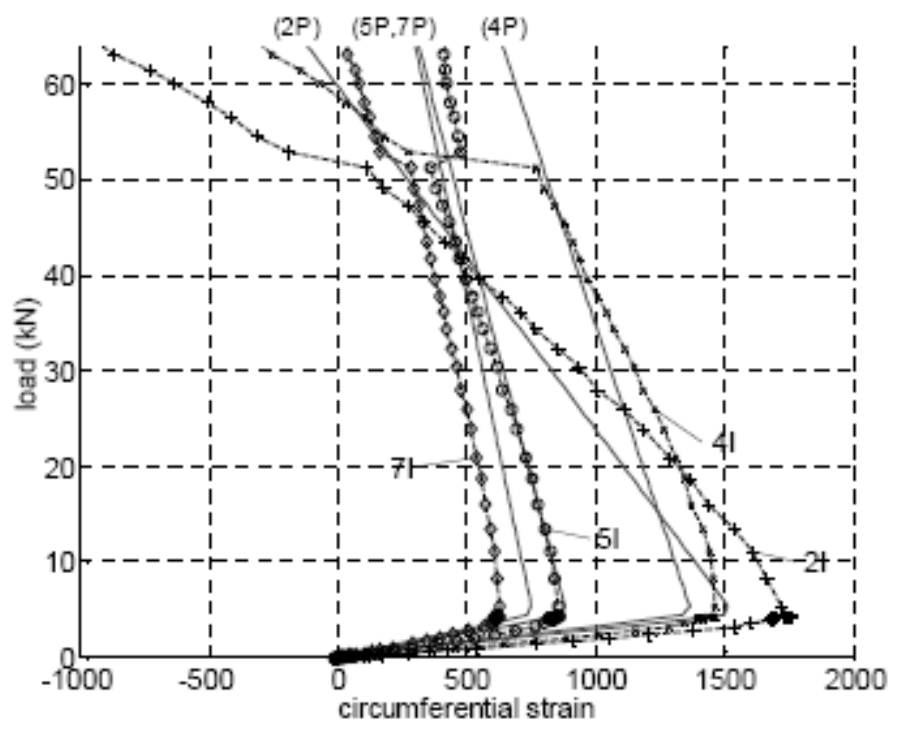

(a)

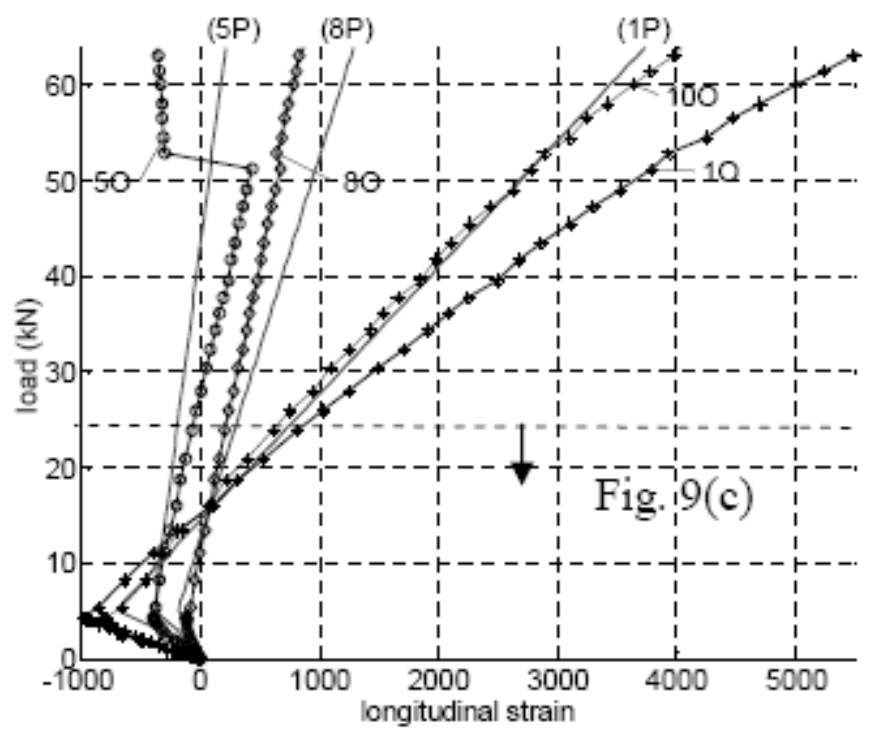

(b)

Figure 11. Pressure/tension behaviour up to $65 \mathrm{kN}$ (a) circumferential strains and (b) longitudinal strains 